\title{
How to avoid ATM frauds by using mobile communications - ideas and implementation
}

\author{
Hristo D. Panayotov PhD \\ Assen Zlatarov University - Burgas, Bulgaria \\ itko59@gmail.com \\ <DOI:10.26821/IJSHRE.8.9.2020.8904 >
}

\begin{abstract}
:
The article gives a brief overview of security issues when withdrawing ATM cash and the main ways of identity theft. An analysis of the main vulnerabilities for confidential debit and credit card information is made. The main drawback is the one-way transmission in the workflow of ATM data along the chain. The main contribution of the article is several new ideas for verifying and sending confidential data to the issuing bank using mobile communication instead of the standard PIN. Three ways to separate the single transmission are discussed in detail - by identification call, SMS from the bank issuer or QR code, as well as the necessary hardware and software upgrades in the client and server part.
\end{abstract}

Keywords: ATM, security, mobile phones, bank cards, skimming, fraud

\section{INTRODUCTION}

In the second decade of this century, fraud involving theft of personal data concerning means of payment debit and credit cards -has increased. If in 2014 official reports reported more than $\$ 1$ billion in losses, by the end of 2018 they had doubled to 1.7 billion, affecting an average of about 15 million users each year. [1] Along with the abuses in the Internet environment, the cases of those related to the withdrawal of funds from ATM (Automatic Teller Machine) banknote withdrawals are also increasing. In the classic fraud related to the theft of means of payment for the purpose of their use for purchases, betting and other services on the Internet, all information is virtual - card number, validity, owner name and CVV (Card Verification Value / Data / Number), i.e. no material carrier of this information is required.

\section{WAYS OF IDENTITY THEFT THROUGH ATM}

ATM theft, the main purpose of which is cash enrichment, requires a material duplicate of the means of payment - imitation of the real card with the magnetic stripe or chip carrier of the basic data. You also need a PIN (Personal Identification Number) known only to the real owner. Detecting a PIN is a much more difficult procedure, unlike CVV (CVT, CVV2), which is most often written on the back of the card. This type of theft is much more difficult than the virtual one, but on the other hand it guarantees a much higher degree of anonymity when draining the money of the rightful owner - millions of ATMs scattered around the world. The tools for their implementation are enriched and innovated with the development of technology, which makes their detection increasingly difficult. The set of tools, techniques and technology in the implementation of this type of fraud is known as skimming. [2]

Skimming is essentially a procedure in which an electronic reader hidden in an ATM scans and stores all the information recorded on the magnetic tape or microchip of the card. A PIN is also required for full access to the bank account. It is usually obtained using microcameras hidden on or near the ATM keyboard. They are positioned so as to obtain a clear picture of the keyboard and record all actions. PIN theft can sometimes be done with audio recording when dialing it. Another popular method is to replace the keyboard with a fake one that records the entire keylog during the operation. [3]With the advent of the first abuses, ATM manufacturers, banking and IT professionals have focused their efforts on improving security techniques and technologies. As early as the early 1990s, card plastics with a microchip appeared, aimed at better protection of personal data from magnetic tape. Basically, information concerning authentication 
Volume 8 Issue 8 August 2020

and authorization of bank transactions is recorded on the chip, which in the case of old cards is recorded on the magnetic tape. However, the introduction of such maps began only at the end of the last century, and for example the United States has been planning for several years to try to switch entirely to this technology. Many other countries have benefited from this innovation and chip cards are already an accepted standard.

In 2006, a team from Massachusetts created a device to read data from RFID (Radio Frequency IDentification) cards, allowing "tap \& go" identification of the payment via wireless connection. The problem later turned out to be precisely that it could be intercepted and read by another external source - a skimmer with a reader close enough to the card. The team continues to improve the technology, introducing the so-called. dummy numbers for encrypted data transmission in a single transaction. The problem here remains with the still insufficient security. However, optimism continues with NFC (Near Field Communication), built into modern smartphones as the future of wireless

\section{payment technologies. \\ 3. ATM WORKFLOW AND SECURTTY DEFICIENCIES}

Figure 1 shows a simplified diagram of the principle of operation of the ATM. Confidential information (customer card data and PIN) is completed using the device reader and its keyboard. The PIN is encrypted and the data is sent to the Host Processor. There the verification of the sent data for the card and the PIN is performed and in case of coincidence the transaction is routed to the cardholder's bank or the institution that issued the card. If the requested service is cash, the host processor initiates an electronic transfer of funds from the client's bank account to the host's receiving account. After the funds are transferred to it, the host sends an approval code to the ATM with permission for the machine to release the amount.

From what has been said so far, several important conclusions can be drawn:

- Despite advances in technique and technology since the introduction of the first maps in the 1950s, abuses have increased and, more recently, exponentially.

- Innovations - RFID and NFC technologies are still under development and fail to become comprehensive, becoming the standard for a number of reasons - technical and social.

- The introduction of the microchip also does not solve the main security problem - card fraud based on this standard continues.

- $\quad$ For the entire nearly 70-year evolution, there has been no change in the technology of data acquisition and transfer. All confidential information - name, validity, card data and PIN are entered from one device (ATM) and transmitted by a single transmission to the host intermediary.

The latter is perhaps the most significant disadvantage of modern ATMs in terms of security. This is also a problem with modern POST (Point of Sale Terminal), as well as in e-commerce using Internet technologies. If until the end of the last century, this way of transmitting confidential information was inevitable, then at the end and especially at the beginning of this century, conditions were created for a real separation of this single transmission.

\section{MOBILE COMMUNICATIONS IN INCREASING THE SECURITY OF ATM}

The ubiquity of Internet technologies, and especially mpbile digital communications, create real preconditions for bypassing some of this confidential data (eg PIN) and completing the entire application only at the host or the bank issuing the card means of payment.

According to estimates by The International Telecommunication Union, GSM subscribers in the world are approaching 7 billion (6.9). This is the equivalent of $95.5 \%$ of the world's population. Of course, there is duplication of subscriptions here, but in any case, they are personally approaching 6 billion [4]. At the same time, the holders of card payments worldwide are: 1.167 billion (Census Bureau) credit card holders and almost twice as many debit card holders - VISA - over 2.3 billion and MasterCard over 1.5 billion. [5] The basic conclusion is selfevident - every debit / credit card holder has a means of mobile communication (the opposite is not certain). This allows the use of a mobile device not only as a means of communication, but also as an opportunity for data transmission or verification when working with an ATM. It should be clarified that these are not smartphones that allow banking transactions via standard Internet channels, but ordinary GSM devices that allow sending data via simple SMS or simple user verification via CLIP (Calling Line Identification Presentation). In recent years, this has become a security standard for most of the banking sector, as well as in other vulnerable areas such as health and social activities. Prior to the advent of smartphones on 
Volume 8 Issue 8 August 2020

the Internet, many M-pay systems operating payments and services based on mobile communication were successfully operating. [6]

The integration of GSM technology for verification in ATM cash operations from the point of view of communication could be realized in the following variants:

- Free call of the client to a number indicated in the ATM menu, receipt of the client's CLIP and disconnection. This method of verification requires prior definition of one or more telephone identification numbers when issuing the payment instrument by the issuing bank. The completed card data + CLIP request is submitted for service to the HOST Processor.Figure 1. Var.1

- The other verification option is more complex, but by no means less reliable. Its choice must initiate the sending of an SMS by the bank - issuer of a randomly generated code to the customer's phone, which manually enters it from the ATM keyboard. Control, long used by many banks in the yerification of 1 5.CONGLUSION Internet payments. Figure 1. Var 22 Security in non-cash payments has long outpaced

- The last idea is not so much related to mobile AfM cash transactions, with the introduction of codes communications, but it has to do with smartphones. It is an individual QR code generated by the issuing bank and stored on the customer's smart device. Instead of entering a PIN, an ATM scanner scans the QR from the customer's phone and forms the request to the HOST Processor. Figure 1. Var.3

- Other verification options with split transmission. In addition to standard GSM communication, other technologies used in modern smartphones can be used. Bluetooth and NFC would also be suitable for transmitting some of the confidential information to the customer without using the vulnerable, theft, ordinary ATM keyboard.

Implementing this much more reliable way of transmitting confidential data instead of entering a PIN code is relatively easy and financially acceptable.

$>$ Hardware - the need for one GSM terminal to each ATM device connected to the computer

\section{REFERENCES:}

[1] "Facts + Statistics: Identity theft and cybercrime", https://www.iii.org/fact-statistic/facts-statisticsidentity-theft-and-cybercrime, accessed May 2020

[2] "How Credit Card Skimming Works", https://www.thebalance.com/, accessed Jan 2020 via a serial port / USB or COM port, depending on the available interfaces /. The prices of such devices are negligibly small, compared to the price of a much more complex ATM device. The terminal and the antenna device to it can be placed in the ATMwithout any design changes. QR code reader integrated in the camera or other location of the ATM.

> Software. The main platforms RMX, OS / 2, Microsoft DOS to Windows, as well as Linux / UNIX in some countries, would allow easy software refinement. Thus, the menu for entering a PIN should be finalized by adding the above-described options - call for CLIP, SMS with verification code from the issuing bank or scan the QR code of the client. The software on the servers of the issuing bank should also be finalized in the part of the verification through the mobile terminals and the $\mathrm{QR}$ verification.

for additional verification, the use of virtual cards, as well as specialized systems such as PayPal, Google wallet, Paysera and many others. At the same time, cash transactions remain vulnerable, despite the measures taken to color banknotes in case of burglary, cameras, anti-skimming measures. The main reason for this is the only point and transmission for performing cash operations. Shared ideas for participating in mobile communications are easy to implement, greatly increasing security. The mass introduction of NFC in modern mobile devices would only facilitate further verification.

Despite the rapid introduction of non-cash payments and especially those in the Internet environment, cash remains the main method of payment for a huge number of debit and credit card holders. Increasing reliability and security would lead to greater confidence in the banking system as a whole and a significant reduction in crime.

[3] "How to Prevent Debit Card

Fraud",https://www.daveramsey.com/blog/debit-cardfraud, accessed Jun 2020

[4] Statistics

,https://www.statista.com/statistics/740154/worldwideunique-mobile-subscribers-by-region/, accessed Jul 
Volume 8 Issue 8 August 2020

\section{0}

[5] Statistics,

https://www.statista.com/statistics/624337/number-ofmastercard-debit-cards-usa-

globally/\#statisticContainer, accessed Jul 2020
[6] Sotirova E., H. Panayotov, M. Krawszak, P. MeloPinto, Modeling of e-trade with mobile communications by the apparatus of generalized networks - In :- Proceedings of the Fifth International Workshop on Generalized Nets, Sofia, 10 Nov. 2004, 41-47

\section{APPENDICES:}
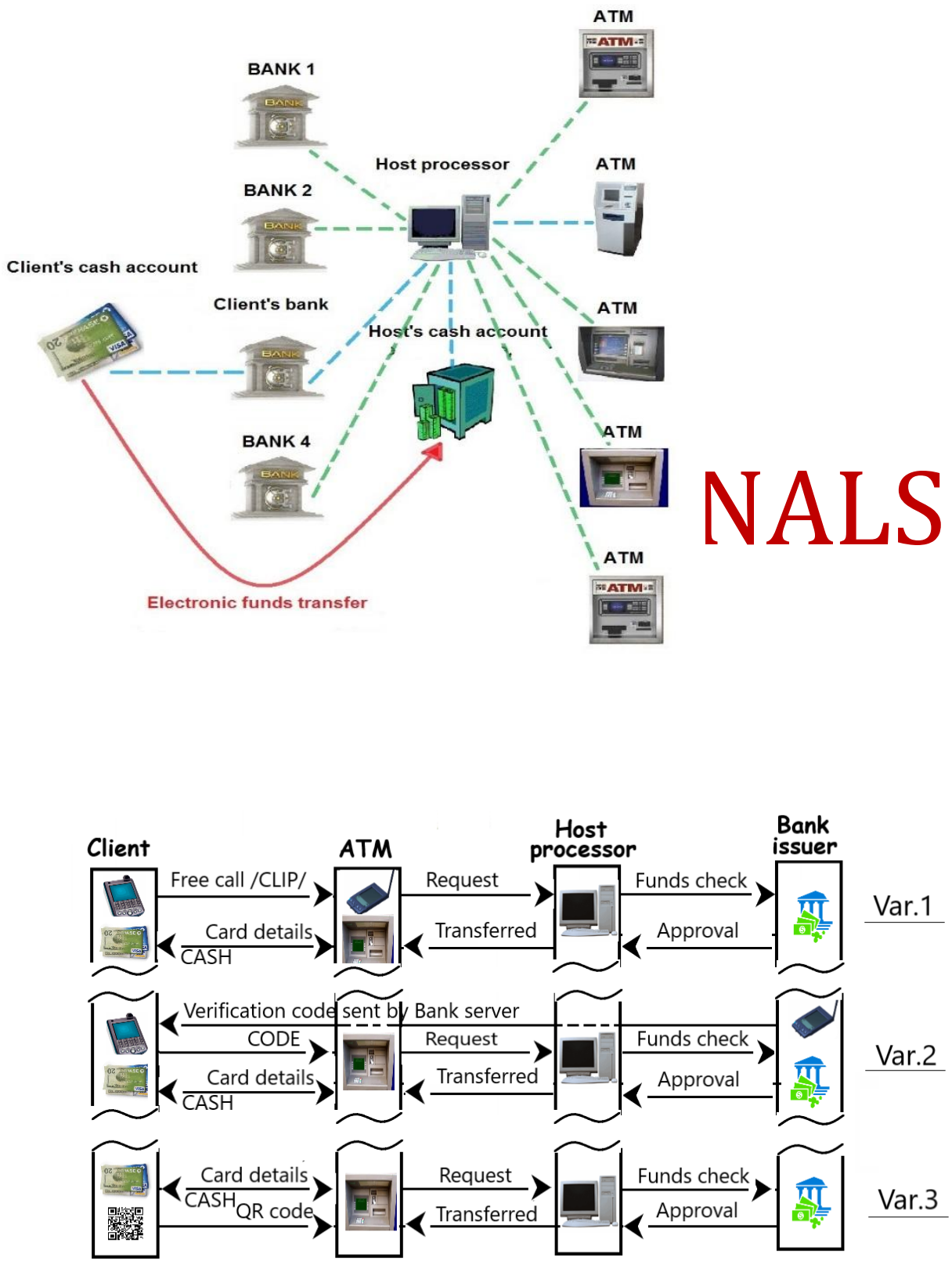

Figure 1. ATM withdrawal activity diagram

Figure 2. ATM workflows using mobile communication 